Relations industrielles

Industrial Relations

\title{
Le rapport de la commission d'enquête sur les relations du travail de la Législature de l'Ontario
}

\section{Gaston Cholette}

Volume 14, numéro 3, juillet 1959

URI : https://id.erudit.org/iderudit/1022288ar

DOI : https://doi.org/10.7202/1022288ar

Aller au sommaire du numéro

Éditeur(s)

Département des relations industrielles de l’Université Laval

ISSN

0034-379X (imprimé)

1703-8138 (numérique)

Découvrir la revue

Citer ce document

Cholette, G. (1959). Le rapport de la commission d'enquête sur les relations du travail de la Législature de l'Ontario. Relations industrielles / Industrial

Relations, 14(3), 379-404. https://doi.org/10.7202/1022288ar

Tous droits réservés @ C Département des relations industrielles de l’Université Laval, 1959
Ce document est protégé par la loi sur le droit d'auteur. L'utilisation des services d'Érudit (y compris la reproduction) est assujettie à sa politique d'utilisation que vous pouvez consulter en ligne.

https://apropos.erudit.org/fr/usagers/politique-dutilisation/ 


\section{COMMENTAIRES}

\section{Le rapport de la commission d'enquête sur les relations du travail de la Législature de l'Ontario}

\section{Gaston Cholette}

Le 27 mars 1957, la Législature de l'Ontario formait une commission chargée d'effectuer une enquête et de faire des recommandations sur tous les aspects de la loi des relations ouvrières ${ }^{1}$ de cette province. Ce comité, composé de onze députés, était présidé par James A. Maloney, de Renfrew South.

Après avoir tenu plusieurs séances publiques au cours desquelles de nombreuses associations lui ont présenté des mémoires, la commission a remis son rapport final ${ }^{2}$ le 10 juillet 1958 , mais celui-ci n'a été rendu public qu'en février 1959, lors de la cinquième session de la vingt-cinquième Législature.

Le document préparé par la commission contient la lettre de dépôt, la liste des membres du comité, un index, une introduction, des remerciements, un exposé de la procédure suivie, un chapitre sur l'arrièreplan historique de la loi des relations ouvrières ontarienne, un résumé de cette loi, des remarques générales sur les principaux problèmes étudiés, les recommandations proprement dites qui forment le corps principal de cet ouvrage, enfin des appendices: a) le rapport intérimaire en date du 25 mars 1958; b) la liste des associations ouvrières, patronales et autres qui ont présenté des mémoires; c) des statistiques; d) une bibliographie.

Pour permettre au lecteur de cette Revue de se faire une idée exacte du rapport de la commission d'enquête de la Législature de l'Ontario, il faudrait en publier ici le texte au complet en regard de la loi qui en fait l'objet. Faute d'espace, il est malheureusement impossible de réaliser ce projet, d'autant plus qu'il faudrait quand même ajouter des commentaires. On doit donc se limiter à quelques aspects seulement du rapport. Le choix des sujets commentés ici a été inspiré par l'importance que la commission elle-même leur a accondée et par l'intérêt que les mêmes problèmes suscitent dans la provinoe de Québec.

(1) The Labour Relations Act, Revised Statutes of Ontario, 1950, Chapter 194, as amended by 1954, Chapter 42;1956, Chapter 35; 1957, Chapter 57, and 1958, Chapter 47 and Rules of Practice and Procedure and Regulations, 1958, Toronto.

(2) Report of the Select Committee on Labour Relations of the Ontario Legislature. 
CoNGÉdIEMENTS POUR ACTIVITÉ SYNDICALE

Dans ses remarques générales, la commission commence par signaler qu'un fort pourcentage des 715 suggestions qu'elle a reçues ont trait au système de conciliation et d'arbitrage. Ce sont en général des critiques s'adressant au Ministère du travail, au Service de conciliation et aux membres des conseils d'arbitrage (Conciliation boards) à cause des délais qui s'accumulent entre la date de la requête pour intervention conciliatrice et celle de la remise des recommandations par le conseil d'arbitrage.

Presque tous les mémoires ouvriers traitent longuement de cette question et proposent les corrections suivantes au système actuel, qui dans ses grandes lignes est semblable à celui de la province de Québec: le stage du conseil d'arbitrage devrait être éliminé ou rendu facultatif et les unions ouvrir̀res devraient avoir le droit de faire la grève 90 jours après l'expiration de la convention collective.

Au début de ses recommandations, la commission affirme presque solennellement que la conciliation et l'arbitrage constituent la pierre angulaire de la loi ontarienne. Pour cette raison, ses membres ont consacré beaucoup de temps à l'étude de ce problème et ont tenu à consulter les statistiques spécialement préparées par le Ministère du travail de l'Ontario sur cette question. ${ }^{3}$ Les recommandations de la commission sur cette matière sont les plus longues de toutes celles que l'on trouve dans le rapport.

Le rapport contient une analyse intéressante des causes de délai. En 1956-57, si l'on tient compte uniquement des 502 cas réglés au stage du conciliateur, on constate qu'il s'est écoulé en moyenne 17 jours entre la date de référence de l'affaire à ces officiers du Ministère du travail et celle de la tenue d'une première séance de conciliation. Dans la plupart des cas, de délai était dû au fait que le représentant patronal ou ouvrier n'était pas disponible pour une période de deux ou trois semaines. Le nombre moyen de jours couverts par le stage du conciliateur dans les 502 cas concernés est de 60 .

Lorsqu'une entente provisoire intervient à la table de négociation, l'union ouvrière demande invariablement un délai pour soumettre les

(3) Time Lapse in Disputes Disposed of by the Conciliation System under the Ontario Labour Relations Aot 1956-1957 and 1955-1956, Ontario Department of Labour, Toronto, May 1958.

Study of Conciliation Board Chairmen: Their Performance and Their Reports, 1955-56 and 1956-57, Ontario Department of Labour, Toronto, June, 1958. Charts Showing Average Time Lapse in Disputes Disposed of by the Conciliation System 1956-1957 under the Ontario Labour Relations Act, Ontario Department of Labour, Toronto, May, 1958.

Statistical Tables on Time Lapse in Conciliation under the Ontario Labour Relations Act, 1956-1957, 1955-1956, Ontario Department of Labour, Toronto, May, 1958. 
propositions à la ratification de l'assemblée générale. Cette procédure exige en moyenne 16 jours, depuis le moment où la séance de conciliation est ajournée jusqu’à la transmission du résultat de la réunion au conciliateur.

Dans 228 cas qui ont franchi les deux étapes de la conciliation et de l'arbitrage, les statistiques indiquent qu'il a fallu en moyenne 157 jours, à partir du moment de la requête pour intervention conciliatrice, pour parvenir au stage de la production du rapport du conseil d'arbitrage. Il faut en moyenne 13 jours à la partie patronale et à la partie ouvrière pour la nomination de leur arbitre respectif; ces deux représentants mettent habituellement 22 jours à choisir un président ou à demander au ministre de le faire à leur place, et enfin il s'écoule en moyenne 26 jours entre la date d'entrée en fonction du président et celle de la première séance du conseil d'arbitrage.

La plupart des mémoires soumis à la commission blâment le président du conseil d'arbitrage pour ce dernier délai, mais il appert, après un examen serré des faits, qu'il est extrêmement difficile, lors de la convocation d'une réunion, de fixer une date qui convienne à tous les intéressés, c'est-à-dire aux membres du conseil d'arbitrage, aux négociateurs patronaux et ouvriers, aux conseillers des employeurs et aux officiers des centrales ouvrières. Lorsqu'une séance d'arbitrage est ajournée, on ne peut presque jamais la reprendre avant deux ou trois semaines car le programme des personnes intéressées est trop chargé. Les mêmes affirmations pourraient être faites au sujet des conseils d'arbitrage de la province de Québec. La commission fait remarquer que dans tous les cas où une extension de mandat a été accordée au conseil d'arbitrage, ce fut du consentement même des deux parties.

La commission refuse d'admettre la prétention selon laquelle la procédure de conciliation et d'arbitrage serait accélérée si l'union ouvrière était libre de faire la grève 90 jours après la date d'expiration de la convention collective. Voici comment la commission arrive à cette conclusion. Elle commence par citer quelques chiffres. Sur les 852 cas référés au Ministère du travail durant l'année fiscale 1956-1957, 502 ont été réglés su stage du conciliateur, 62 n'ont pas été soumis à l'arbitrage à cause d'une recommandation du conciliateur à cet effet, 8 ont été retirés au stage du conciliateur, 51 ont été réglés avant la première séance d'arbitrage, 93 ont été réglés par le conseil d'arbitrage au cours de l'instruction de l'affaire, 135 ont fait l'objet d'une recommandation arbitrale, enfin 1 a été retiré.

D'après l'examen de ces faits, la commission souligne tout d'abord l'excellent résultat du travail des conciliateurs. En plus d'avoir disposé avec succès de 502 différends, ces officiers ont participé à la solution des 62 conflits à propos desquels le ministre n'a pas jugé opportun de constituer un conseil d'arbitrage, et enfin ils ont contribué au règlement des 51 cas qui, après le stage du conciliateur, n'ont pas atteint l'étape de la première séance du conseil d'arbitrage. Il est 
bon de noter que la position du conciliateur est singulièrement renforcée en Ontario du fait qu'il peut refuser de recommander la formation d'un conseil d'arbitrage.

Quant aux conseils d'arbitrage, ils ont effectué un accord entre les parties idans 93 cas et ont rédigé des rapports dans 135 autres. Or seulement 19 de ces 135 conflits ont dégénéré en grèves, ce qui, dans l'esprit des membres de la commission, prouve que les recommandations des conseils d'arbitrage ont sans doute été très utiles dans la plupart des cas. Ce n'est pas si sûr que cela.

En fait certaines unions ouvrières, notamment celles qui représentent les travailleurs de l'industrie de l'automobile, traitent les conseils d'arbitrage comme des institutions encombrantes et n'attachent pas d'importance ou même d'attention à leurs rapports. Dans la province de Québec on remarque des attitudes semblables dans les secteurs où les syndicats ouvriers ont une grande puissance économique. Au lieu de présenter une documentation abondante et une preuve testimoniale élaborée aux arbitres, ces syndicats cherchent à brûler les étapes et à réduire au strict minimum ce qu'ils considèrent comme la formalité du stage du conseil d'arbitrage. Dans certains cas, ils se bornent à soumettre leurs demandes sans les justifier ou encore, à l'extrême, ils retirent leurs revendications afin que le conseil d'arbitrage produise immédiatement son rapport.

En général cependant, et surtout dans la petite industrie, les syndicats ouvriers font tout leur possible pour impressionner favorablement les arbitres afin d'obtenir d'eux des recommandations avantageuses. Il va sans dire que cette dernière attitude est généralisée dans le secteur des services publics où la sentence du conseil d'arbitrage est obligatoire et finale. Sur ce point la situation est différente en Ontario car dans cette province les ouvriers ont droit de faire la grève après l'arbitrage.

Après ces constatations, la commission affirme que si le mécanisme de la conciliation et de l'arbitrage avait cessé automatiquement de fonctionner au 90ième jour de l'expiration de la convention collective, aucun des 228 cas qui sont passés par l'arbitrage en 1956-57 n'aurait pu être terminé à ce stage. La commission en tire la conclusion quaucune recommandation arbitrale n'aurait pu être faite, que dans chaque cas la compagnie aurait sûrement refusé de continuer les négociations car elle aurait été sous une menace de grève, et qu'enfin les 228 différends se seraient envenimés au point que la plupart d'entre eux auraient dégénéré en grèves.

Je ne crois pas pour ma part que cette conclusion est fondée. Tout ici ne peut être que supposition et hypothèse, car on ne saura jamais ce qui se serait produit dans un contexte différent. La commission a tort de conclure que si la grève devenait légale 90 jours après l'expiration de la convention, aucun des 228 conflits référés à l'arbitrage en 1956-57 n'aurait pu franchir complètement les stages de la 
conciliation et de l'arbitrage. Raisonner ainsi revient à faire abstraction de l'acoélération très probable que l'éventualité plus rapprochée d'une grève aurait sans doute imprimée au mécanisme des négociations à toutes les étapes. La commission cherche à se confirmer dans une opinion préconçue par une opération rétrospective trop simple qui équivaut à une interprétation abusive des faits. On peut toutefois considérer l'attitude de la commission comme valable dans un nombre de cas plus ou moins grand.

Une deuxième conclusion de la commission me paraît inexacte et exagérée. Si la date du droit à la grève survenait pendant l'arbitrage que ce soit 90 jours ou plus après l'expiration de la convention - il ne s'ensuivrait pas automatiquement la suspension de tout dialogue et encore moins la rupture définitive des négociations. Dans bien des cas au contraire les négociations en deviendraient rapidement plus sérieuses. Un grand nombre d'employeurs ne demanderaient pas mieux que de classer sans tarder des négociations qui leur dévorent un temps si précieux.

Enfin on ne saura jamais si effectivement il y aurait eu 228 grèves en Ontario au lieu de 19 en 1956-57 si en fait les conseils d'arbitrage n'avaient pas eu le temps de terminer leurs travaux. Il est pour le moins tout aussi plausible de supposer que dans la très grande majorité des cas le conflit se serait terminé sans grève. Dans cette dernière hypothèse, il est important de souligner que les différends auraient duré beaucoup moins longtemps, ce qui donnerait au système ainsi conçu une supériorité incontestable sur le régime actuel.

Chose certaine, c'est que si les deux parties trouvent leur profit dans l'arbitrage - ce qui devrait normalement être le cas - il n'y a aucune raison pour que l'une ou l'autre rompe brusquement les pourparlers du seul fait que le délai légal requis pour l'exercice du droit de grève serait écoulé. La commission de la Législature admet ellemême d'ailleurs, à un moment donné, que les deux parties ont toujours consenti, en fait, à l'extension du délai du conseil d'arbitrage lorsque celui-ci n'avait pas eu le temps de terminer son oeuvre. La commission se servait alors de cet argument pour confondre ceux qui se plaignent des lenteurs de l'arbitrage.

Dans ses remarques générales, la commission déclare que le système de conciliation et d'arbitrage actuellement en vigueur en Ontario est substantiellement satisfaisant. Son but, disent les membres de la commission, est d'aider les parties à s'entendre à l'amiable, et même si cela entraîne des délais de cinq ou six mois, le jeu en vaut certainement la chandelle si ce moyen s'avère efficace pour éviter des grèves. Cependant, un des membres de la commission, le représentant de York South, est d'avis que l'on devrait tenter l'expérience du recours facultatif à la conciliation et à l'arbitrage.

Je trouve que la commission d'enquête de la Législature de l'Ontario fait un piètre plaidoyer en faveur du régime actuel. Elle donne 
nettement l'impression qu'elle veut à tout prix garder subtantiellement intacte la présente structure de l'intervention gouvernementale dans le règlement des conflits d'intérêts. Les arguments qu'elle emploie ne sont pas convaincants et plusieurs d'entre eux vaudraient tout autant pour la défense d'un système d'arbitrage volontaire. La commission ne fait aucune allusion à l'expérience de pays comme les Etats-Unis et la Grande-Bretagne qui ont un régime beaucoup plus libéral sous ce rapport que celui de l'Ontario et elle ne compare pas les résultats obtenus dans cette province avec ceux de ces pays. Le refus de la suggestion qui maintiendrait le caractère obligatoire du stage du conciliateur mais qui rendrait facultatif le recours à l'arbitrage n'est pas motivé. En somme, même si on était d'accord sur les conclusions de la commission d'enquête, on ne pourrait pas se servir de son rapport pour défendre efficacement son point de vue et réduire les critiques au silence.

La commission fait une série de suggestions destinées à réduire les délais dans la conciliation et l'arbitrage et de nature à améliorer la qualité des services rendus par les personnes impliquées dans ces procédures. Elle recommande au Ministère du travail de faire un effort concerté dans le but de recruter un plus grand nombre de sujets qui seront capables, après une période d'entraînement, d'agir comme conciliateurs et même d'être promus ensuite au poste de présidents de conseils d'arbitrage. En plus de recevoir un entraînement pratique, la commission ajoute que les conciliateurs et les présidents de conseils d'arbitrage devraient posséder des qualités spéciales qui ont trait à la personnalité, au tempérament, au caractère, etc.

D’après la commission, le Ministère du travail devrait nommer un officier de personnel qui serait spécialement chargé de recruter des candidats sérieux. Ces candidats devraient ensuite suivre des cours en relations industrielles aux frais du gouvernement dans une université ou une institution d'enseignement spécialisée dans ce domaine. Enfin, certains d'entre eux pourraient agir comme secrétaires de conseils d'arbitrage ou comme asssitants de présidents de tels conseils, ce qui compléterait leur formation.

Je suis d'avis que la commission a parfaitement raison d'insister sur la nécessité d'un sérieux recrutement de bons candidats au poste de conciliateur. Le personnel actuel en Ontario se compose en majorité d'officiers qui avaient d'abord occupé d'autres fonctions au sein du Ministère du travail. Quelques autres ont été recrutés dans les cadres du mouvement ouvrier ou des entreprises. Dans l'ensemble ils s'acquittent bien de leur tâche.

Il n'y a aucun doute que le conciliateur doit avoir des qualités toutes spéciales sous le rapport de la personnalité, du caractère et du tempérament. Il lui faut de l'ascendant et le don d'inspirer confiance et respect, sans quoi il devient le jouet des parties ou un intrus que l'on tolère mal. Mais cela ne suffit pas. Il a besoin également d'une formation académique qui lui permette d'être de niveau au moins 
égal à celui des professionnels de la négociation qu'il coudoie presque quotidiennement, et il doit posséder des outils de travail au moins aussi bons que ceux des négociateurs patronaux et ouvriers. Dans ces conditions, il serait normal que le salaire du conciliateur se compare favorablement à celui des négociateurs patronaux et ouvriers. La commission de la Législature de l'Ontario insiste avec beaucoup d'à propos sur l'importance de cours adéquats. Elle aurait pu ajouter des recommandations sur la nécessité d'adjoindre au service de conciliation une équipe compétente de chercheurs et une abondante documentation.

La commission recommande que le Ministère du travail forme un personnel de présidents de conseils d'arbitrage par le recrutement direct et par la promotion des conciliateurs les plus qualifiés. Cette suggestion n'est pas très claire. La commission reconnaît d'ailleurs elle-même que si cette suggestion a quelque mérite, elle ne serait pas d'application facile. On ne sait pas si la commission songe à la constitution d'une nouvelle catégorie de fonctionnaires ou tout simplement à une rationalisation du système actuel qui consisterait à mettre plus d'ordre dans le choix d'arbitres à temps partiel.

L'idéal serait que syndicats ouvriers et employeurs se forment euxmêmes les arbitres dont ils ont besoin au lieu de s'en remettre au gouvernement. Aux Etats-Unis l'initiative privée réussit à merveille dans ce domaine. Des centaines de personnes y exercent le métier d'arbitre de carrière en relations du travail. Ils sont choisis par les compagnies et les unions ouvrières sans que l'Etat n'ait à s'en mêler. La plupart d'entre eux ne sont pas de la profession légale, car l'arbitrage privé s'est développé en marge du légalisme et en réaction contre lui.

En Ontario, plus de la moitié des personnes désignées pour agir comme présidents de conseils d'arbitrage sont des juges. " Ils s'acquittent fort bien de leur tâche, puisque les parties aux différends suivent elles-mêmes cette tendance, mais la somme des travaux que l'arbitrage leur impose les distrait de leur fonction principale. La commission de la Législature de l'Ontario a donc raison de proposer le recrutement et la formation systématiques de personnes qui auront pour rôle propre la présidence de conseils d'arbitrages en matière de relations industrielles.

Dans le but d'accélérer la marche de l'arbitrage, la commission d'enquête propose que le Ministère du travail désigne une ou plusieurs personnes dont la tâche serait de vérifier constamment l'état des travaux de chaque conseil d'arbitrage et de faire la chasse aux délais inutiles. Il est possible que l'adoption de cette recommandation ap-

(4) Cf. Study of Conciliation Boand Chairmen: Their Performance and Their Reports, 1955-56 and 1956-57, Ontario Department of Labour, Toronto, June 1958. 
porte une certaine amélioration, mais il est plutôt probable que ces fonotionnaires deviendraient tout simplement des pointeurs ou joueraient le rôle de la mouche du coche. En effet, même si la marche de chaque conseil d'arbitrage était confiée à la surveillance d'un fonctionnaire, celui-ci ne pourrait jamais éliminer l'obstacle permanent qui gêne le fonctionnement du mécanisme, c'est-à-dire l'impossibilité pour la plupart des personnes impliquées d'être facilement disponibles.

La rémunération actuelle des membres des conseils d'arbitrage n'est pas suffisante pour favoriser le recrutement d'un personnel qualifié. La commission recommande donc que les honoraires du président soient portés de $\$ 60$ à $\$ 75$ par jour, et ceux des autres membres, de $\$ 20$ à $\$ 50$ par jour. Dans la province de Québec, ces taux sont respectivement de $\$ 30$ et de $\$ 20$ par jour.

Enfin, la commission recommande que le conseil d'arbitrage fasse rapport au ministre dans les guinze jours de la fin de l'audition, à moins que les parties ne soient d'accord sur une période plus longue. Cette suggestion est très importante, car un conseil d'arbitrage met quelquefois beaucoup plus de temps à délibérer et à rédiger ses recommandations qu'il en a fallu aux parties pour présenter leur preuve. Dans la plupart des cas, ces délais sont attribuables aux nombreuses autres occupations des membres des conseils d'arbitrage ou tout simplement à leur négligence.

\section{Arbitrage des griefs}

La loi ontarienne stipule que toute convention collective doit contenir une clause comportant le règlement obligatoire par arbitrage de tout grief, y compris un différend sur la question même de l'arbitrabilité. Si les parties omettent de se conformer à cette prescription, la convention contient ipso facto la clause-type insérée dans la loi. Cette clause prévoit la formation d'un conseil d'arbitrage ad hoc composé de trois membres. Lorsque l'arbitre patronal et l'arbitre syndical ne réussissent pas à choisir un président, c'est le Ministre du travail qui le désigne.

Sans entrer dans les détails, on peut dire que ce régime ressemble beaucoup à celui de la loi québecoise, avec cette importante différence qu'en Ontario les conciliateurs n'interviennent pas dans le règlement des griefs et que dans le Québec un arrêt de travail est permis lorsque les procédures d'arbitrage sont terminées et qu'un délai de quatorze jours s'est écoulé.

La commission d'enquête ne consacre pas beaucoup d'espace au problème de l'arbitrage des griefs mais elle fait à ce sujet quelques recommandations importantes. Elle suggère tout d'abord que la clause-type prévue dans la loi soit corrigée en y ajoutant que l'arbitre n'aura pas le droit de modifier en aucune façon le texte de la conven- 
tion collective. Il s'agit là d'une mesure de protection opportune conter la fantaisie possible d'un arbitre, car celui-ci n'a pas pour but de négocier à la place des parties mais de prononcer des jugements dans le cadre de la convention existante. Cette précaution est déjà prise d'ailleurs dans la plupart des conventions elles-mêmes en Ontario et aux Etats-Unis.

Afin de donner plus de poids aux sentences arbitrales, la commission propose que celles-ci soient rendues exécutoires sous l'autorité de la Commissoin de relations ouvrières et que de sévères punitions soient imposées à ceux qui refuseraient de $s^{3} y$ conformer. Les décisions devraient être rendues dans les quatorze jours qui suivent l'audition à moins qu'un consentement écrit à l'effet contraire ne soit adressé par les parties au greffier de la Commission de relations ouvrières. La commission est d'avis que les arbitres devraient avoir les mêmes pouvoirs que les conseils d'arbitrage (conciliation boands) chargés des conflits d'intérêts.

La commission considère que les sentences arbitrales ne devraient plus être l'affaire exclusive des parties puisqu'elle recommande qu'elles soient rendues par écrit et déposées au Ministère du travail où n'importe qui pourrait les consulter lorsqu'elles auront été classées et cataloguées. C'est là une très heureuse suggestion car ces décisions constitueraient une jurisprudence fort utile.

Dans la province de Québec, un grand nombre de sentences sont publiées par le Ministère du travail car la plupart des arbitrages se font sous ses auspices. Malheureusement ces documents ne sont pas publiés sous une forme qui en rende la consultation facile et rapide. Vu qu'une tendance à l'arbitrage privé se dessine très nettement, surtout dans les secteurs où les entreprises et les syndicats ouvriers sont de taille imposante, on possède de moins en moins de documentation arbitrale. Il est à souhaiter que le Québec et l'Ontario finissent par faire aussi bien que l'initiative privée aux Etats-Unis, car dans ce pays le Bureau of National Affairs publie et catalogue de façon satisfaisante les principales sentences arbitrales. ${ }^{5}$

Enfin la commission revient sur l'importance de former des arbitres compétents. Les commentaires qui ont été faits sur ce point en ce qui concerne les différends de négociation sont encore plus pertinents dans le domaine du règlement des griefs.

Il est curieux de constater qu'au moment où l'initiative privée dans la province de Québec a tendance à se prévaloir de plus en plus de la liberté qu'elle a de régler elle-même les griefs, la commission d'enquête ontarienne se prononce en faveur d'une intervention gouvernementale croissante. Dans la province de Québec, les compagnies

(5) Labour Arbitration Reports, The Bureau of National Affairs, Inc., Washington 7, D.C. 
et les syndicats qui ont institué l'arbitrage privé n'ont aucune relation avec le gouvernement dans le règlement de leurs différends relatifs à l'interprétation et à l'application de la convention collective. Ils choisissent habituellement eux-mêmes à l'avance leurs arbitres et ils ne sont astreints à aucune disposition impérative de la loi.

En Ontario, au contraire, si les recommandations de la commission étaient aoceptées, le gouvernement exigerait le dépôt des sentences, contrôlerait les délais dans le prononoé des jugements, définirait le statut des arbitres et se chargerait de l'organisation de la profession d'arbitre ou d'une catégorie de fonctionnaires qui agiraient comme présidents de conseils d'arbitrage.

Toutefois, si on comparaît aotuellement les deux provinces sous le rapport du degré d'intervention gouvernementale dans le règlement des griefs, on découvrirait probablement que le Québec a la prépondérance. Cela tient surtout au fait que le Ministère du travail québecois défraie les honoraires et les dépenses du président et qu'il délègue un conciliateur auparavant afin de tenter d'effectuer un accord à l'amiable. Au surplus, l'arbitrage privé sans recours au Ministre du travail pour la désignation du président est apparemment plus répandu en Ontario que dans le Québec.

La commission ontarienne, qui s'est pourtant inspirée largement de l'expérience des Etats-Unis et notamment de la loi Taft-Hartley ${ }^{\circ}$ sur d'autres questions, aurait pu tirer plusieurs leçons avantageuses de l'exemple américain dans le règlement des griefs. La loi américaine n'interdit pas la grève comme mode de règlement des griefs. C'est l'initiative privée qui s'est volontairement chargée de le faire. Presque toutes les conventions stipulent en effet que l'arbitrage sera l'étape finale de la procédure. Des centaines d'individus agissent comme arbitres sous les auspices d'associations privées et environ 250 d'entre eux, groupés dans la National Academy of Arbitrators, sont de véritables professionnels de l'arbitrage. Le gouvernement se contente de tenir au point une liste d'arbitres qu'il désigne seulement à la demande des parties sans encourir de frais. Si le système américain a ses lacunes et ses équivoques, qu'il n'y a pas lieu d'étudier longuement ici, il constitue par ailleurs un magnifique témoignage en faveur de linitiative privée. C’est la leçon qư'il importe de retenir.

INRUSTRIE DE LA CONSTRUCTION

Après la conciliation et l'arbitrage, ce sont les problèmes de l'industrie de la construction qui sont traités le plus longuement dans le rapport de la commission d'enquête de la Législature de l'Ontario. En Ontario comme dans la province de Québec, l'application de la législation ouvrière soulève de nombreuses difficultés dans le domaine de la construction.

(6) Labor-Management Relations Act, 1947, Public Law 101, 80th Congress, Cliapter 120, lst Session, N.R. 3020. 
Les unions ouvrières en Ontario se plaignent de ne retirer aucun avantage de la loi des relations ouvrières et demandent en conséquence d'être tout simplement dégagées des obligations de cette loi. Les ouvriers prétendent que les unions de métiers, à cause de leur nature même, s'ajustent mal au mécanisme de certification de cette loi; ils soutiennent qu'un grand nombre de chantiers de construction sont terminés avant que la certification ou la conciliation aient eu lieu, enfin ils soulignent que les conflits ont été plus nombreux au cours des dernières années qu'avant l'adoption de la loi.

Les associations patronales, d'autre part, sont d'avis que les clauses de sécurité syndicale en vigueur dans les conventions collectives de l'industrie de la construction ont permis aux unions de métiers d'acquérir beaucoup de puissance. Elles déplorent que les négociations soient devenues de plus en plus difficiles, que les dates d'expiration varient de métier à métier et de région à région, que les querelles de juridiction intersyndicales soient fréquentes et que les arrêts de travail qui s'ensuivent soient gravement préjudiciables aux entrepreneurs. Afin de remédier à ces désordres, elles proposent l'abolition de la Trade Unions Act, ce qui aurait pour effet de rendre les unions ouvrières vulnérables devant le pouvoir judiciaire; elles suggèrent en outre que les unions soient assujetties à un régime de permis afin de les obliger à respecter les lois provinciales; enfin elles demandent que de sévères sanctions soient imposées aux unions ouvrières dans les cas d'infractions.

Il y a des similitudes entre l'Ontario et le Québec en ce qui concerne les difficultés d'aocréditation. Celles-ci proviennent surtout du caractère passager des chantiers de construction et de la grande fluctuation du nombre des ouvriers à l'emploi du même entrepreneur.

Dans la province de Québec, la Commission de relations ouvrières n'accorde pas beaucoup de certificats dans ce domaine: vu que la plupart des chantiers ont une existence inférieure à douze mois, les parties seraient dans l'impossibilité de négocier une convention d'une durée minimum d'un an, ce qui, d'après la Commission, irait à l'encontre des prescriptions de l'article 15 de la loi des relations ouvrières (ch. 162A). La Commission accorde un certificat seulement si elle prévoit que les travaux de construction dureront assez longtemps pour être régis par une convention d'une, de deux ou de trois années. Dans le cas d'ouvriers permanents travaillant pour un entrepreneur en particulier dans la tuile et le terrazo, la plomberie, l'électricité et le posage du fer structural, la Commission accorde des certificats aux unités locales de chacun de ces corps de métiers. L'unité de négociation ainsi délimitée ne comprend pas les ouvriers temporaires mais elle vaut pour les réguliers dans toute la juridiction territoriale de la province. Pour les autres corps de métiers, la politique de la Commission est différente, car elle exige leur amalgamation avant de les accréditer comme agents négociateurs à l'égard d'un entrepreneur particulier. 
En Ontario le nombre des certificats est beaucoup plus élevé que dans le Québec. En 1955-56 et en 1956-57, la Commission de relations ouvrières d'Ontario a aocordé 211 et 223 certificats dans le domaine de la construction, ce qui représente respectivement $24.6 \%$ et $21.0 \%$ de tous les certificats émis au cours de ces années. Dans la province de Québec, il y a tout au plus une centaine de certificats de la Commission en existence.

Une des principales raisons de cette différence, en dehors de l'importance relative du volume de la construction dans les deux provinces, c'est l'existence du régime des décrets dans la province de Québec. Grâce à cette formule les syndicats ouvriers ne sont pas obligés de négocier une multitude de conventions, ce qui les dispense de rechercher la certification dans la plupart des cas. On verra un peu plus loin que certaines des recommandations de la commission d'enquête de la Législature de I'Ontario visent en fait à édifier dans cette province une structure de négociation qui s'apparente beaucoup à celle qui existe dans le Québec. Ici en effet les conditions de travail établies conjointement et volontairement par des parties qui ont une importance prépondérante dans leur branche d'activité et dans une région déterminée, font l'objet d'un décret ministériel qui les extensionne obligatoirement aux tiers, stabilisant et uniformisant ainsi les rapports de travail dans de vastes régions et même dans certains cas, par exemple en ce qui concerne les ascenseurs, à travers toute la province. En 1957-58, sur un total de 28,056 employeurs et de 262,867 ouvriers qui étaient assujettis à des décrets, il y avait 11,227 employeurs et 142,087 ouvriers de l'industrie de la construction. ${ }^{7}$

Les difficultés que les syndicats ouvriers éprouvent au sujet de la certification dans les deux provinces, surtout dans les gros chantiers, les poussent parfois à la violence. Ici dans le Québec le problème de la reconnaissance syndicale a été l'une des causes prédominantes de certaines grèves fameuses qui sont encore fraîches à la mémoire de tous: Chute-des-Passes, Haute-Rive, etc. Dans chacun de ces cas et dans beaucoup d'autres, même lorsqu'il n'y avait pas de grève, le Ministre a chargé le Service de conciliation et d'arbitrage de procéder à létablissement du caractère représentatif des syndicats impliqués, et il a ensuite fait émettre par ce service un certificat qui comporte pour l'employeur une obligation morale de négocier. En pratique, et compte tenu des circonstances, cette méthode a donné d'assez bons résultats car elle s'accompagnait d'une conciliation spéciale où le prestige du Ministère du travail était en jeu et dans laquelle celui-ci injectait une forte dose de pression morale afin de valoriser son certificat.

En Ontario, la commission d'enquête propose une méthode différente. Sa principale recommandation, c'est la formation d'une section

(7) Rapport général du Ministre du travail de la province de Québec, 1958, p. 184. 
spéciale au sein de la Commission de relations ouvrières pour l'industrie de la construction. Théoriquement, une solution orientée dans cette voie semble plus normale que le double système actuellement en vigueur dans la province de Québec. En Ontario, la Commission ne donne pas une interprétation restrictive à la disposition de la loi concernant la durée minimum d'un an des conventions collectives.

Sur les autres questions relatives à l'industrie de la construction, la commission d'enquête semble avoir été débordée. Elle repousse carrément la principale revendication du monde ouvrier en disant qu'il ne saurait être question de soustraire une partie des syndicats de travailleurs aux obligations que la loi impose. Cette attitude est valable; mais lorsque la commission propose un réaménagement des structures existantes elle ne trouve pas de solution précise. Voilà pourquoi ellesuggère la convocation par le Ministère du travail d'un congrès conjoint de la construction qui réunirait autour d'une même table les. représentants des quelque 25 associations patronales et d'un nombre à peu près égal d'unions ouvrières qui existent en Ontario dans cette industrie.

L'ordre du jour de cette réunion serait le suivant:

1. Formation d'une fédération patronale provinciale et d'une fédération ouvrière provinciale ayant le pouvoir de négocier des conventions générales.

2. Des conventions générales dans le cadre du métier, de la région ou de la province.

3. Une convention générale couvrant tous les métiers (sur le modèle de la convention de l'Hydro).

4. Des dates uniformes d'expiration de toutes les conventions collectives.

5. Une procédure spéciale et rapide de certification adaptée à l'industrie de la construction.

6. Une procédure spéciale de conciliation et d'arbitrage adaptée à l'industrie de la construction.

7. Une revision de la Industrial Standards Act.

8. La constitution d'un organisme conjoint chargé de régler les conflits de juridiction dans l'Ontario.

9. La nomination d'un groupe spécial de commissaires au sein de la Commission de relations ouvrières pour accélérer le mécanisme d'accréditation.

On voit que la commission d'enquête de la Législature de l'Ontario a préféré laisser à un autre groupement le rôle principal dans la recherche de formules nouvelles. Elle a toutefois le mérite d'en avoir indiqué quelques grandes lignes par le truchement de l'ordre du jour proposé.

Il n'y a aucun doute que la structure des négociations dans le Québec, grâce au régime de la loi de la convention collective (ch. 163), est beaucoup mieux adaptée que celle de l'Ontario à l'industrie de la construction. En effet les buts recherchés par la commission d'enquête ontarienne à travers les item $1,2,3$ et 4 sont en grande partie déjà atteints dans la province de Québec au moyen des déorets et des comités paritaires. Quant aux querelles de juridiction, elles sont ici beaucoup 
moins nombreuses, probablement à cause des comités paritaires et de l'existence de syndicats nationaux qui ne reçoivent pas de directives de centrales ouvrières situées dans un autre pays.

A mon avis, si les ouvriers de la construction doivent être astreints aux obligations de la loi des relations ouvrières, ce qui est normal, il faut à tout prix accélérer la procédure d'accréditation, de conciliation et d'arbitrage dans leur cas particulier. Autrement, la plupart d'entre eux ne bénéficieraient pas des avantages de la loi.

En fait, peu importe la législation, les unions ouvrières dans la construction jouiront toujours cependant d'un statut spécial qui est la meilleure garantie de leur sécurité. Dans cette industrie comme dans celle de limprimerie, on est en présence de forces qui s'exercent surtout sur le plan local ou régional. Un grand nombre de petites entreprises y fait régner une concurrence très vive dans un champ géographique plutôt restreint. Les employeurs ont donc besoin d'une réglementation et, forcément, ils doivent accepter le fait syndical et se rendre aux exigences des syndicats ouvriers dans le domaine de la sécurité. Il s'agit là de besoins si impérieux et si bien ressentis de part et d'autre que dix ans d'excommunication de l'atelier fermé par la loi Taft-Hartley aux Etats-Unis n'ont pas entamé la forteresse que patrons et ouvriers ont érigée et continuent à maintenir conjointement autour de cette forme de protection syndicale. ${ }^{7} \mathrm{a}$

Commission de relations ouvrières (Ontario Labour Relations Board)

Avant mars 1958, la Commission de relations ouvrières de l'Ontario se composait d'un président, d'un vice-président, de deux membres représentant les employeurs et de deux autres représentant les employés. Mais depuis cette date l'article 66 (2a) autorise le gouvernement à nommer un plus grand nombre de commissaires représentant et les employeurs et les travailleurs. La Commission peut siéger en deux bancs différents pourvu qu'il y ait quorum dans chaque cas.

Malgré cela, la commission d'enquête est d'avis que la Commission de relations ouvrières, telle que constituée, est surchargée de travail. Elle propose que le gouvernement soit autorisé à désigner d’autres commissaires neutres. Elle suggère aussi que les représentants ouvriers proviennent d'un grand nombre d'organisations de travailleurs afin que la représentation ouvrière soit aussi large que possible. Elle croit que le président et le vice-président devraient être considérés comme représentants du public en général.

Il semble que la présence de commissaires représentant les employeurs et d'un nombre égal d'autres membres représentant les em-

(7a) Cf. The Taft-Hartley Act After Ten Years: A Symposium, Industrial and Labor Relations Review, Volume II, no 3, avril 1958, pp. 352-353-354; Cornell University, Ithaca, N.Y. 
ployés soit bien agréée dans les milieux patronaux et ouvriers de l'Ontario. Le même phénomène se constate pour le Conseil canadien des relations ouvrières, qui comprend un président, un vice-président et un nombre égal de membres représentant les employés et les employeurs.

Dans la province de Québec, la Commission de relations ouvrières ne compte aucun membre qui représente statutairement les patrons et les ouvriers. Aucun d'entre eux, en fait, n'a été choisi dans les rangs du monde patronal. L'expérience d'Ottawa et de Toronto fournit un argument très sérieux à la Confédération des travailleurs catholiques du Canada, à l'Association professionnelle des industriels et à d'autres associations patrrinales et ouvrières qui réclament des changements dans la composition de la Commission de relations ouvrières québecoise.

La commission d'enquête fait des commentaires au sujet de la question des décision écrites. Elle signale que des représentations lui ont été faites dans le but d'obliger la Commission de relations ouvrières à motiver par écrit toute décision, déclaration ou règlement. Elle constate tout d'abord que c'est la pratique courante de la Commission de relations ouvrières d'agir ainsi lorsqu'elle prend des décisions importantes, et elle l'engage fortement à continuer dans cette voie. Elle recommande ensuite que la politique des décisions motivées par écrit reçoive une application aussi large que possible, sans toutefois proposer que la Commission de relations ouvrières doive être astreinte à cette exigence dans tous les cas. Enfin, la commission d'enquête recommande que toutes les décisions écrites soient déposées au Ministère du travail et deviennent accessibles au public sur demande. Toutes ces suggestions correspondent à un voeu souventes fois exprimé dans la province de Québec au sujet des décisions de la Commission de relations ouvrières de cette province. L'ouvrage publié récemment par le secrétaire de la Commission québecoise est une amorce dans la bonne direction. ${ }^{8}$

La commission d'enquête recommande à la Législature qu'il y ait un droit d'appel de toutes les décisions de la Commission de relations ouvrières, sur autorisation du juge-en-chef de l'Ontario, qu'il s'agisse de questions de droit seulement, de questions de fait ou des deux ensemble. Il est regrettable que la commission d'enquête ne précise pas quelle cour aurait juridiction pour entendre ces appels. Sur ce point il y a eu dissidence de la part de deux membres de la commission. Ceux-ci désirent le statu quo, c'est-à-dire le maintien du caractère final des décisions de la Commission de relations ouvrières.

Il s'agit ici d'une question fort discutable. Les partisans du statu quo peuvent soutenir avec raison que l'on doit interdire le droit d'appel afin de conserver au travail de la Commission de relations ouvrières

(8) G. Vanllancourt, Recueil annoté des lois ouvrières, Wilson et Lafleur, Ltée, éditeurs, Montréal, 1951. 
son caractère expéditif. Ils ajoutent d'ailleurs que l'on peut toujours en appeler d'une décision de la Commission si l'on prétend que celle-ci a outrepassé sa juridiction. Par contre, ceux qui réclament le droit d'appel invoquent l'importance des décisions de cet organisme administratif qui exerce des fonctions judiciaires et quasi-judiciaires.

Il serait peut-être opportun d'autoriser l'appel des décisions de la Commission de relations ouvrières, mais alors il faudrait accorder à cette procédure une priorité sur' d'autres affaires judiciaires et la limiter à une seule instance. Agir autrement ouvrirait la porte à toutes sortes de procédés dilatoires contraires à l'intérêt des parties et à celui du public en général.

Afin de renforcer l'autorité de la Commission de relations ouvrières, la commission d'enquête propose que ses décisions soient homologuéeś par la Cour supérieure et assimilées à un jugement de cette Cour. De plus, les amendes prévues pour refus de se soumettre aux décisions de la Commission de relations ouvrières devraient être plus élevées. Je crois que ces suggestions sont excellentes.

Certification ou accréditation

L'article $7(2)$ de la loi des relations ouvrières stipule que si $45 \%$ au moins et $55 \%$ au plus des ouvriers dans l'unité de négociation font partie du syndicat requérant, la Commission de relations ouvrières devra tenir un vote pour tirer l'affaire au clair. Après le scrutin, la Commission accorde un certificat si la majorité des ouvriers ayant droit de vote se prononcent en faveur de leur union.

La commission d'enquête remarque qu'avec ce procédé, les ouvriers qui ne votent pas ou qui refusent de le faire se prononcent en fait contre le syndicat; elle signale aussi qu'elle a reçu des demandes visant à faire prévaloir dans ce domaine les principes électoraux traditionnels des démocraties politiques, ce qui veut dire que l'accréditation devrait être accordée dès qu'il se dégage une majorité chez ceux qui votent effectivement.

La commission d'enquête est d'avis que la Commission de relations ouvrières devrait organiser un vote lorsqu'elle découvre qu'au moins $35 \%$ et au plus $75 \%$ des ouvriers dans l'unité de négociation font partie du syndicat. Le seul cas où il y aurait certification automatique sans tenue d'un scrutin serait celui où plus de $75 \%$ des travailleurs de l'unité de négociation sont membres du syndicat et à condition qu'il n'y ait aucune contestation à ce sujet.

Pour ma part, je crois que ce dernier pourcentage est trop élevé. Le vote ne devrait être obligatoire que pour dissiper des doutes. Lorsque la Commission de relations ouvrières, après enquête, constate que 
les effectifs syndicaux représentent de $45 \%$ à $55 \%$ du total de l'unité de négociation, il est normal qu'elle oblige l'association requérante à établir ses titres au caractère représentatif au moyen d'un vote, surtout si une demande à cet effet lui est présentée par l'employeur ou par une autre union ouvrière intervenante. Mais il est exagéré de soumettre le syndicat à cette formalité lorsque des inspecteurs gouvernementaux ont déjà vérifié les effectifs et constaté que $75 \%$ des ouvriers du collège électoral sont des membres réguliers de leur syndicat.

Lorsqu'un vote est pris, la commission d'enquête recommande que la Commission de relations ouvrières émette un certificat si plus de la moitié des voteurs sont en faveur du syndicat, pourvu que les $2 / 3$ des membres du collège électroral participent au scrutin, ou encore si plus de la moitié de ceux qui ont droit de vote se déclarent en faveur du syndicat. La commission suggère enfin que la date qui devrait être retenue pour l'établissement des effectifs syndicaux en regard du nombre de travailleurs dans l'unité de négociation, soit celle de la mise à la poste de la requête, pourvu que l'envoi soit recommandé, et non la date de réception du document par la Commission de relations ouvrières.

Si la recommandation de la commission d'enquête concernant l'émission d'un certificat à la suite d'un vote était acceptée, il suffirait à l'extrême que plus de $331 / 3 \%$ des membres du corps électoral votent en faveur du syndicat pour que celui-ci soit accrédité. Cette hypothèse ne se réaliserait, bien entendu, que si $662 / 3 \%$ seulement des ouvriers dans l'unité de négociation exerçaient leur droit de vote.

L'attitude de la commission ontarienne marque donc une étonnante évolution des esprits. Elle réjouira sans doute les milieux ouvriers mais elle se heurtera sûrement à de puissantes résistances dans le monde patronal. A bien y réfléchir, on se rend compte que la recommandation de la commission ne fait que transposer dans un secteur particulier ce qui se pratique déjà sur le plan politique. Avec le système de scrutin majoritaire à tour unique des lois électorales canadiennes, un parti politique peut théoriquement prendre le pouvoir avec lappui de moins du tiers des électeurs qui ont droit de vote. Plusieurs rétorqueront que le pouvoir politique est indispensable dans la société civile, tandis que la présence d'un syndicat ouvrier n'est pas nécessaire dans toutes les entreprises. Il reste qu'il est normal d'envisager l'adoption de mesures plus libérales dans l'accréditation des syndicats ouvriers, car autrement on perpétuerait des restrictions de moins en moins acceptables à l'exercice du droit naturel d'association. Ces changements devraient toutefois entraîner la disparition de certaines tactiques repréhensibles que certains agents ouvriers utilisent fréquemment dans les ‘ampagnes d’organisation syndicale. 


\section{SF́CURYTÉ SYNDICALE}

La commission commence par signaler que dans le passé la sécurité syndicale était un sujet très litigieux au cours des négociations, surtout lorsqu'il s'agissait de la perception des cotisations syndicales par l'employeur (check-off). Les représentants patronaux disaient que la sécurité syndicale n'était pas une oondition de travail, qu'ils n'étaient pas obligés de financer les syndicats ouvriers et qu'enfin la retenue obligatoire des cotisations était une violation de la liberté des ouvriers.

En Ontario la mentalité patronale a évolué graduellement, si bien qu'à l'heure actuelle 95\% des conventions collectives en vigueur dans cette province pourvoient à un mode quelconque de retenue des cotisations. Toutefois, il reste encore des compagnies qui refusent de s'engager dans cette voie en dépit des recommandations des conseils d'arbitrage. Les employeurs qui ont pris cette position ne changeront de conduite que si la loi les y oblige.

En terminant ses remarques générales sur cette question, la commission informe le public que de très fortes pressions ne sont exercées pour que la sécurité syndicale demeure une affaire à régler autour de la table de négociation. La commission est toutefois d'opinion que le temps d'apporter une solution d'ordre législatif à ce problème est désormais arrivé. Elle recommande donc que la loi des relations olvrières contienne une disposition rendant obligatoire l'adoption d'une clause de retenue syndicale volontaire et révocable dans les conventions collectives, aux conditions suivantes:

a) La majorité des ouvriers dans l'unité de négociation devront d'abord se prononcer en faveur de cette mesure au scrutin secret.

b) Le syndicat devra fournir à l'employeur des autorisations écrites à cet effet signées par la majorité des travailleurs dans l'unité de négociation.

c) La clause deviendra nulle et de nul effet dès que les révocations auront fait tomber le nombre des autorisations à moins de la moitié des employés dans l'unité de négociation.

d) La retenue des cotisations ne s'appliquera que dans le cas des employés qui auront signé une autorisation à cet effet.

La commission signale la dissidence de trois de ses membres sur cette question de retenue syndicale. Ces derniers ne veulent aucune intervention législative dans ce domaine.

Dans la province de Québec, il semble exister une tendance semblable à celle que la commission ontarienne a observée. Des statistiques compilées en 1953 indiquaient que $75.4 \%$ des conventions alors en vigueur contenaient une clause de retenue syndicale. ${ }^{9}$ Il y a de fortes raisons de présumer que cette proportion a augmenté par la suite, surtout depuis que le pouvoir judiciaire a constaté le caractère

(9) Cf. Problèmes d'autorité au sein de l'entreprise, dixième congrès des relations industrielles de Laval, Québec, 1955, pp. 33-34. 
légal de la retenue syndicale volontaire et révocable. ${ }^{10} \mathrm{La}$ Cour suprême du Canada vient même de déclarer légale la version courante de la «formule Rand» qui comporte la retenue obligatoire des cotisations syndicales ou de leur équivalent lorsqu'une disposition à cet effet existe dans une convention collective. Mais si cette forme de retenue est désormais légale, sa généralisation est loin d'en être automatiquement assurée et le débat sur son mérite reste ouvert pour longtemps.

Quant à l'atelier syndical et à l'atelier fermé, la commission d'enquête a été frappée par le fait que dans certains cas des organisateurs syndicaux veulent forcer des employeurs à accepter ces mesures de sécurité syndicale lorsqu'en fait aucun travailleur dans l'unité de négociation ne fait partie du syndicat ouvrier. La commission recommande donc que ces formules ne soient autorisées que dans le cas de syndicats aocrédités ou si l'union ouvrière impliquée peut prouver qu'au moment de la signature de la convention elle représentait une majorité suffisante de travailleurs (plus de $75 \%$ ) pour avoir droit à la certification automatique.

A l'heure actuelle, l'article 33 de la loi ontarienne établit un régime presque semblable à celui du code national du travail. ${ }^{12}$ Les parties à une convention collective ont donc le droit d'insérer une clause stipulant, comme condition d'emploi, la qualité de membre d'un syndicat ouvrier spécifié, ou acoordant une préférence d'emploi aux membres de tel syndicat. L'attitude de la commission d'enquête marque ainsi un durcissement au sujet de l'atelier syndical et de l'atelier fermé. La commission n'a pas cru opportun cependant d'aller jusqu'à proposer que la loi ontarienne s'inspire sur ce point de la loi Taft-Hartley telle qu'elle a existé de 1947 à 1951. Au cours de cette période, la loi américaine subordonnait l'adoption des clauses de sécurité syndicale sauf l'atelier fermé qui était prohibé et qui le demeure encore - à l'approbation préalable de la majorité des travailleurs. Les succès remportés par les unions ouvrières dans ces scrutins et l'extension généralisée de la pratique de l'atelier syndical ont convaincu le législateur que les dispositions restrictives qu'il avait adoptées n'avaient plus leur raison d'être. Depuis octobre 1951, la loi ne soumet plus la négociation des clauses de sécurité syndicale non interdites à la tenue d'un vote affirmatif préalable chez les ouvriers. On peut dire que la recommandation de la commission d'enquête ontarienne constitue une excellente mesure de contrôle de la volonté ouvrière sans gêner inutilement l'action des syndicats majoritaires.

Dans la province de Québec, la négociation de beaucoup de conventions collectives serait grandement facilitée si la loi était clarifiée

(10) Cf. Price Brothers Company Limited vs Pierre Letarte \& Syndicat national des travailleurs de la pulpe et du papier de Riverbend Inc. \& Al., B.R., 1953, p. 312 .

(12) 11-12 Geo. VI, chap. 54, 1948, art. 6. (Loi sur les relations industrielles et sur les enquêtes visant les différends du travail.) 
par intervention législative au chapitre de la liberté et de la sécurité syndicales. Il se fait encore une dépense énorme de temps, de paroles et d'énergie à la table de négociation sur le caractère légal ou illégal d'un grand nombre de mesures de sécurité syndicale. Il serait donc opportun, non pas d'obliger l'employeur à aocepter une clause de sécurité syndicale, mais de faire expressément de cette matière, avec les réserves voulues, une chose négociable.

La commission ontarienne a été fortement impressionnée par les difficultés qu'éprouve un ouvrier qui perd son emploi parce qu'il a cessé d'être membre d'un syndicat ouvrier, lorsque la raison de sou exclusion n'est pas le refus de payer les cotisations. D'après la constitution des unions ouvrières, le travailleur ainsi congédié peut en appeler à un tribunal syndical supérieur mais cette procédure est longue et difficile. Il est malheureusement arrivé des faits qui justifient ces craintes de la commission.

La commission d'enquête hésite à faire des recommandations sur cette question, car dès le début elle s'est tracée comme ligne de conduite de ne pas intervenir dans les affaires internes des unions ouvrières. Toutefois, sous réserve de la dissidence de deux de ses membres, elle propose que la loi accorde un droit d'appel devant la Commission de relations ouvrières à tout ouvrier congédié pour la raison qu'il aurait cessé d'être membre du syndicat ouvrier, sauf si le motif de son départ est le refus de payer ses cotisations. Cette recommandation ne sappliquera pas si lunion ouvrière a établi un tribunal spécial en dehors de ses rangs pour disposer rapidement de cette sorte d'affaires. Pour ma part, je crois que la proposition de la commission, avec les nuances et les réserves qu'elle comporte, est justifiée.

\section{CONGÉdiements POUR ACTIVITÉ SYNDICALE}

La commission signale que des employeurs auraient fréquemment recours à des pratiques interdites, par exemple des congédiements ou la fermeture d'une partie de l'établissement, lors de campagnes d'organisation syndicale. L'article 57 de la présente loi stipule que le Ministre du travail, lorsqu'il reçoit une plainte à ce sujet, peut désigner un enquêteur et lui demander un rapport. Il semble que beaucoup de porte-parole ouvriers se sont plaints de l'inefficacité du système actuel de protection du droit d'association au cours de la période d'organisation d'un syndicat ouvrier dans une entreprise.

On trouve dans le rapport de la commission d'assez longues recommandations sur ce point. La commission suggère d'abord que toute demande d'enquête dans pareil cas soit faite à la Commission de relations ouvrières. Lorsque celle-ci est d'avis qu'il y a lieu de donner suite à la requête, elle demande au Ministre de désigner un conciliateur qui est chargé d'effectuer une enquête et de tenter de trouver une solution au différend. 
Jusqu'ici la procédure suggérée est semblable à celle qui a été longuement pratiquée dans la province de Québec. Depuis une couple d'années, cependant, la Commission de relations ouvrières a décidé de confier cette tâche à ses inspecteurs. Ceux-ci sont bien en mesure d'accomplir leur travail si on limite leur rôle à celui d'un enquêteur, mais dès qu'il s'agit de tenter d'effectuer un aocond il serait préférable de déléguer un conciliateur.

La commission ontarienne suggère que le conciliateur fasse rapport à la Commission de relations ouvrières. $\mathrm{Si}$ aucune des parties ne contestait le rapport du conciliateur dans les quatorze jours, la Commission de relations ouvrières serait libre de prendre la décision qui lui paraîtrait la plus appropriée, notamment: le rejet de la plainte, la réintégration de l'ouvrier congédié à son emploi avec ou sans remboursement du salaire perdu. Si le rapport du conciliateur était contesté, la Commission de relations ouvrières nommerait un enquêteur qui aurait les mêmes pouvoirs qu'un conseil d'arbitrage. La Commission rendrait ensuite un jugement destiné à appliquer les recommandations de l'enquêteur. Dans certains cas spéciaux, la plainte pourrait être entendue par la Commission de relations ouvrières elle-même.

Si la Législature de l'Ontario donnait suite à ces suggestions, il n'y a aucun doute que le droit d'association serait effectivement protégé surtout pendant les campagnes d'organisation syndicale. Le seul moyen légal d'empêcher un employeur de couper les têtes d'un syndicat naissant, c'est de l'obliger à reprendre les officiers qu'il aurait congédiés et à leur payer le salaire perdu.

La commission d'enquête est toutefois d'opinion que l'employeur devrait avoir un droit égal à celui des syndicats de parler librement aux ouvriers, à condition de ne pas recourir à la contrainte, à lintimidation, aux menaces, aux promesses ou à d'autres procédés d'influence indue. Il n'y a pas de doute que la commission s'est inspirée de la loi Taft-Hartley en faisant cette recommandation.. Deux de ses membres sont dissidents car ils préfèrent s'en tenir à l'article 45 de la loi actuelle, en tenant compte de l'interprétation et de l'application que lui ont données la Commission de relations ouvrières. Cet article stipule que nul employeur n'a le droit de participer ou de faire obstacle à la formation ou à l'administration d'un syndicat ouvrier, ou encore de lui accorder une assistance financière ou morale.

Dans la province de Québec, une partie faible de la loi est précisément celle qui a trait aux congédiements pour activité syndicale. Même si le fardeau de la preuve repose sur l'employeur, des présidents de conseils d'arbitrage obligent en pratique le plaignant ou le syndicat à démontrer le bien-fondé de leur plainte et acceptent facilement de faibles prétextes comme une cause suffisante de congédiement. Une telle anomalie est particulièrement susceptible de se produire dans l'esprit d'un «civiliste». Non seulement la preuve de congédiement 
pour activité syndicale est-elle difficile à faire - car il y a mille façons de tuer un chat - mais encore la seule pénalité prévue pour une telle offense est insignifiante car il s'agit d'une amende de cent dollars $(\$ 100)$. C'est vraiment une aubaine pour un employeur qui veut se débarrasser d'un individu gênant. Il est vrai que des infractions subséquentes peuvent entraîner l'imposition d'une amende de $\$ 1,000$, mais ce n'est pas encore suffisant. En fait, lorsqu'un individu et un syndicat doivent d'abord obtenir de la Commission de relations ouvrières une autorisation écrite de poursuivre et s'engager ensuite dans le dédale des procédures judiciaires, le combat cesse faute de combattants car à la fin il y a beaucoup de chances pour qu'il ne reste plus de syndicat.

Enfin la commission ontarienne déplore que des organisateurs ouvriers se soient vu refuser l'entrée sur le terrain de compagnies en dehors des heures de travail, uniquement parce qu'ils soccupaient d'affaires syndicales. Ces incidents se produisent ordinairement là où les ouvriers sont logés dans des camps, notamment dans l'industrie minière et forestière. Le même phénomène se produit dans la province de Québec. La commission d'enquête avertit les intéressés que si la situation ne change pas, le gouvernement devra légiférer pour mettre fin à ces tactiques.

\section{Piquetage et GRève}

Dès qu'elle touche au piquetage et à la grève, on a l'impression que la commission d'enquête a les nerfs à fleur de peau. Au tout début de ses remarques générales elle rappelle avec insistance les prohibitions de l'article 366 (1) du code criminel qui a trait à l'intimidation, à la violence et aux menaces. Selon la commission, le piquetage tel qu'il est pratiqué couramment est souvent illégal; malgré cela la police et les autorités civiles hésitent à intervenir. Des représentations lui ont été faites pour que la Commission de relations ouvrières déclare le piquetage une pratique interdite et pour que la Commission ait le pouvoir d'intervenir rapidement et efficacement s'il se produisait.

La commission recommande que le piquetage soit interdit dans les cas suivants:

a) Lorsque la Commission de relations ouvrières, en vertu de l'article 59 de la loi, a déclaré qu'une grève est illégale.

b) Lorsqu'il s'agit d'une grève de reconnaissance syndicale.

c) Lorsqu'il s'agit d'une grève de juridiction.

d) Lorsque l'employeur n'est pas directement impliqué dans le différend.

Enfin la commission suggère que le piquetage n'affecte que les travailleurs qui sont dans l'unité de négociation impliquée dans le litige. La commission ontarienne aimerait que le vote de grève ne se prenne que lorsqu'il peut légitimement s'exercer. C'est tout à fait normal si l'on veut éviter des pressions injustifiées. La commission souhai- 
te en outre que le vote de grève ne puisse se prendre qu'une fois que tous les membres de l'unité de négociation auront été dûment informés de la date, de l'heure et de l'endroit où il sera tenu. Elle ne dit pas cependant si le droit de vote appartiendrait uniquement aux membres du syndicat accrédité. Je crois que cette recommandation est opportune, mais elle le serait davantage si elle ne prenait pas place dans un cadre et un contexte qui la font apparaître comme une restriction additionnelle à l'exercice d'un droit déjà très ligoté.

On voit que la commission d'enquête fait écho aux nombreuses plaintes qui s'élèvent un peu partout en Amérique du Nord contre la grève et la plupart des formes de piquetage. La loi Taft-Hartley aux Etats-Unis s'est déjà engagée dans cette voie. La Législature de la Colombie Britannique, au cours de sa dernière session, a adopté une loi qui contient des dispositions de cette nature. Il n'y a aucun doute que le piquetage et la grève, dans bien des cas, provıquent une irritation croissante dans l'opinion publique, surtout quand ils découlent d'une querelle de juridiction et de reconnaissance ou lorsqu'ils sont une manifestation de boycottage indirect.

Il s'agit là d'un des problèmes les plus délicats, les plus difficiles et les plus importants de la législation ouvrière. Le spectre de la grève a toujours provoqué l'antipathie des milieux patronaux, se qui s'explique facilement, et l'intervention des pouvoirs publics au nom de l'ordre et du bien commun. Au fond, presque toute la législation en matière de relations du travail au Canada a pour principale raison d'être le conditionnement du droit de grève. Ce qui est difficile, c'est de concilier l'exercice «pacifique» de ce droit avec les exigences de son efficacité comme arme de la lutte ouvrière.

La commission de la Législature de l'Ontario ne fait aucune concession à une tendance qui s'affirme dans les milieux ouvriers et dans quelques cercles de l'enseignement universitaire en faveur de l'élargissement de l'exercice du droit de grève. ${ }^{13} \mathrm{La}$ commission cherche au contraire à le circonscrire davantage, à le cerner en quelque sorte et à lui dresser un enclos bien serré où le combat puisse se dérouler comme en champ clos sans faire trop de tapage ou trop de dégâts. Encore une fois on retrouve l'influence de la loi Taft-Hartley qui s'est évertuée sans succès à contenir artificiellement la réalité qu'est le phénomène de la grève tout en admettant le principe dont il découle naturellement, c'est-à-dire le système de la libre négociation collective.

Nul ne pourra jamais, surtout sur ce point précis de la législation ouvrière, proposer une solution parfaite. Ce n'est pas ici, dans le cadre d'un commentaire, le moment de s'étendre sur cette question complexe. Mais il me paraît opportun de faire quelques suggestions.

(13) Cf. Opinions de Guillaume Geoffrion, Gérard Picard et H.D. Woods dans Le règlement des conflits d'intérêts en relations du Travail dans la province de Québec, Presses Universitaires Laval, Québec, 1958. 
Ce qui importe avant tout, c'est que les lois en matière de grève soient réalistes. Il semble que le législateur, lorsqu'il s'avance sur ce terrain, se laisse guider par des considérations idéalistes de paix et d'ordre qui sont souvent la négation même de l'exercice efficace du droit de grève. Celui-ci est pourtant reconnu et considéré comme une conséquence directe du principe de la libre négociation collective. ${ }^{14}$ Ce dernier principe lui-même n'est jamais mis en cause dans les pays industrialisés de tradition démocratique.

Le régime actuel en Ontario, comme dans la province de Québec et partout ailleurs en Amérique du Nord, est radicalement équivoque. Il reconnaît la grève mais nadmet pas, juridiquement, les seules formes de piquetage qui soient réalistes lorsque la loi respecte en même temps le droit au travail des travailleurs dissidents ou étrangers i l'entreprise. Il faudrait prendre une position nette: ou bien on libéralise l'exencice du droit de grève tout en maintenant le droit au travail et alors le pouvoir public doit se préparer à combattre la violence physique et les désordres extérieurs en exerçant avec vigueur et sévérité un rôle policier; ou bien, si on conditionne rigoureusement l'exercice du droit de grève, le législateur devrait alors, une fois que toutes les conditions posées pour son exercice sont réalisées, en favoriser l'exécution efficace en supprimant à partir de ce moment le droit au travail ou en acoondant un statut privilégié au droit de grève. Dans cette dernière alternative, il y aurait lieu que le pouvoir public prenne les dispositions nécessaires pour vérifier la volonté majoritaire des ouvriers intéressés, avant et pendant la grève.

Contrôle des AFFAIres des SYNDichts

Les membres de la commission d'enquête de la Législature de l'Ontario ont puisé beaucoup d'éléments dans la loi américaine TaftHartley. $\mathrm{Vu}$ qu'un grand nombre d'unions ouvrières en Ontario ont des affiliations américaines, il était normal que l'on cherche à les assujettir à un certain contrôle semblable à celui qu'elles connaissent aux Etats-Unis. Toutefois, on ne va pas aussi loin que le législateur américain dans cette direction: on ne vise que les fonds de bien-être et de pension administrés par des unions ouvrières.

La commission fait les recommandations suivantes:

1. Les états financiers des fonds de bien-être et de pension administrés par un syndicat ouvrier seul ou en collaboration avec un employeur ou par l'intermédiaire d'une société de fiducie devraient être déposés au Ministère du travail.

2. Les unions ouvrières devraient rembourser à l'ouvrier ses contributions à un fonds de bien-être ou de pension si celui-ci cesse d'être membre pour quelque raison que ce soit ou s'il change d'emploi.

3. Les fonds de bien-être et de pension devraient être utilisés intégralement au Canada.

(14) Cf. ibid., opinion d'Adolf Sturmthal. 
4. Toutes les unions ouvrières devraient fournir chaque année à leurs membres un rapport financier.

Ces suggestions sont opportunes car elles aideront le mouvement ouvrier à se protéger contre les abus qui peuvent s'y glisser, comme partout où d'importantes sommes d'argent sont administrées. Il est bon de noter la recommandation d'utiliser intégralement au Canada les fonds de pension et de bien-être des unions ouvrières, car elle reflète une tendance croissante au nationalisme dans l'opinion anglo-canadienne.

CoMmission D'ENQUÊTE EN MATIÈRE INDUSTRIELLE

A la fin de son rapport, la commission d'enquête de la Législature de l'Ontario fait une recommandation qui reproduit presque textuellement l'article 56 du Code national du travail. La seule différence qu'il convient de mentionner entre les deux, c'est qu'en Ontario le lieutenantgouverneur en conseil serait autorisé, lors de conflits susceptibles d'affecter l'intérêt public, à décréter que toute grève sera interdite aussi longtemps qu'il n'aura pas formé une commission d'enquête industrielle et qu'il n'aura pas reçu de rapport de cette commission.

La Commission d'enquête ne donne pas de détail sur la composition de ces commissions industrielles ni sur leurs pouvoirs, sauf que le gouvernement leur donnerait dans chaque cas un mandat exprès. Elles auraient apparemment, comme dans la loi fédérale, les mêmes pouvoirs que les conseils d'arbitrage. Elles devraient faire rapport dans les quatorze jours de leur formation, à moins que le gouvernement ne leur acconde un délai. Les résultats de l'enquête et les recommandations de la commission industrielle seraient transmis aux parties intéressées et publiées par le gouvernement.

Les pouvoirs qui seraient ainsi attribués au gouvernement ontarien correspondent, grosso modo, ̀̀ ceux que la Loi du Département du travail et la Loi des différends ouvriers de Québec aocordent au Ministre du travail. Il semble que la principale explication de ces recommandations, c'est l'exemple de la loi Taft-Hartley dans la mesure où celle-ci suspend le déclenchement de grèves nationales pendant qu'une enquête se poursuit. Ces commissions d'enquête en matière industrielle auraient peut-être pour effet de rééditer et de doubler les conseils de conciliation et d'arbitrage déjà prévus dans le système de l'Ontario; elles alourdiraient sans l'améliorer le système existant de règlement des conflits.

Cette attitude de la commission d'enquête de la Législature de l'Ontario semble procéder d'une psychose de la grève et d'une mentalité «Ligne Maginot». Les institutions qu'elle prévoit ont leur raison d'être évidente aux Etats-Unis, où le recours à la conciliation et à 
l'arbitrage est facultatif, mais elles prendraient l'allure en Ontario d'une sorte de dernière tranchée, d'un instrument de réserve pour une super-mise en échec dans le but de protéger la société contre le fléau d'une grève.

Cette attitude est d'autant plus surprenante que la commission d'enquête de la Législature, par ailleurs, recommande d'enlever le droit de grève aux employés d'hôpitaux et à tous les travailleurs qui ont la responsabilité d'assurer le fonctionnement de services publics indispensables.

\section{Les caisses de retraite - Nouvelle source de capital}

\section{J. Alasco}

L'étude de la situation du patronat en face du problème des caisses de retraite demande un examen critique de certaines idées qui, tout en étant généralement acceptées, ne sont que rarement analysées de près.

Un coup d'oeil sur les négociations de conventions collectives permet de découvrir la tendance suivante: en effet, quand il s'agit des caisses de retraite, le travail organisé recourt au concept de «dépréciation humaine» pour justifier ses demandes à cet égard. Les dirigeants unionistes ou syndicaux maintiennent que, tout comme l'employeur pourvoit, dans la comptabilité, à la dépréciation des machines d'une entreprise, ainsi faut-il tenir compte de la dépréciation humaine, celle de l'ouvrier engagé dans la production.

C'est là une justification assez habile, avancée vraisemblablement pour des raisons de stratégie ou de tactique, mais sans conviction sincère le plus souvent, et destinée surtout à utiliser un argument de nature sociale ou morale afin d'impressionner l'opinion publique et de la faire pencher d'un côté plutôt que de l'autre.

Du moins est-ce là ce qui se dégage des affirmations et des déclarations des chefs ouvriers. Intéressante et impressionnante même, à première vue, cette idée semble être surtout l'argument rationnel destiné à justifier les caisses de retraite devant l'opinion publique. II me paraît donc opportun d'y consacrer une attention toute particulière et de voir si, oui ou non, cet argument est suffisamment solide pour être valable.

Qu'est-ce que la dépréciation? Elle s'exprime de deux façons différentes. D'abord, il y a la dépréciation physique: c'est l'usure matérielle de l'équipement de production. Ensuite, il y a la dépréciation technologique, c'est-à-dire l'infériorité croissante d'un équipement par rapport à l'équipement plus moderne installé dans des entreprises concurrentes. 